Tmed_48

\title{
Lifestyle monitoring as a predictive tool in telecare
}

Julienne Hanson ${ }^{1}$, Dorota Osipovič ${ }^{1}$, Nick Hine ${ }^{2}$, Telmo Amaral ${ }^{2}$, Richard Curry ${ }^{3}$ and James Barlow ${ }^{3}$

1 Bartlett School of Graduate Studies, University College London, UK

2 School of Computing, University of Dundee, UK

3 Tanaka Business School, Imperial College London, UK

Correspondence:

Professor Julienne Hanson

Bartlett School of Graduate Studies

UCL Faculty of the Built Environment

Torrington Place Site

Gower Street

London WC1E 6BT

UK

(Fax: +44 207916 1887; Email: j.hanson@ucl.ac.uk) 


\section{Summary}

Six people with multiple health problems living in an extra care housing scheme for older people with vision impairment agreed to take part in a telecare trial. An average of 14.8 sensors was installed in each of the flats. The monitoring period began in January 2006 and lasted for 10 months. The data acquired by the sensors installed in each flat was transmitted from the home unit to a central computer. Four interviews were conducted with each participant. Halfway through the study we created a number of case studies of sensor activity at the time of known events in the lives of the participants, together with an attempt to interpret these patterns of activity with the benefit of available contextual information. Although our investigation showed that sensors are capable of identifying some changes in daily routines at the time of important events, the interpretation of such changes requires a large amount of contextual information and the involvement of participants themselves. Various technical and operational difficulties will need to be resolved before it will be possible to use lifestyle monitoring predictively.

\section{Introduction}

Living independently in a home of one's own is an objective for most older people. We have investigated lifestyle monitoring devices, sometimes referred to as third generation telecare.[1] Lifestyle monitoring sensors monitor people's domestic routines and daily activities such as movement around the house, bed and chair occupancy, the opening of cupboards, doors, fridges and wardrobes, and use of electrical devices such as kettles, TVs and lamps. Users are not required to wear any equipment. The idea of lifestyle monitoring is that by constant passive monitoring of the domestic environment, it will be possible to learn people's habits and then to recognise significant deviations from the norm. The ultimate aim of lifestyle monitoring is to provide more timely intervention in the event of an emerging crisis.

\section{Methods}

Six people with multiple health problems living in an extra care housing scheme for older people with vision impairment agreed to take part in the telecare trial. There were four women and two men. At the time of the first interview four participants were in their eighties and the remaining two were in their sixties and forties. Five of the subjects had vision impairment. All of them had other serious health conditions such as hearing impairment, diabetes, heart problems, high blood pressure, poor balance, epilepsy, cerebral palsy and cancer. Five participants lived alone and one participant lived with her husband and a guide dog.

A detailed floor plan of each flat, including the position of the furniture, was prepared in order to help position the sensors. An average of 14.8 sensors was installed in each of the flats, see Table 1. The monitoring period began in January 2006 and lasted for 10 months. The data acquired by the sensors installed in each flat was transmitted from the home unit to a central computer.

Four interviews were conducted with each participant. The main aim of the initial, preinstallation interview was to collect contextual information about participants' daily routines and activities. The second interview took place about six weeks after the 
telecare equipment had been installed in order to record participants' early experiences of the service.

Halfway through the study a data triangulation exercise was undertaken in order to bring together the output from different data streams - house plans, furniture layouts, sensors, health vital signs, domestic routines and information on various past events. This allowed us to create a number of vignettes which represented case studies of sensor activity at the time of known events in the lives of the participants, together with an attempt to interpret these patterns of activity with the benefit of available contextual information. An example vignette is discussed below.

The third interviews were conducted to discuss the vignettes that we had developed in order to record any changes to individual behaviour that could be observed in the pattern of sensor firings in people's flats. The final interviews took place shortly after de-installation of the sensors. In addition, all six participants received regular blood pressure monitoring and two participants received blood glucose monitoring, performed by the housing scheme staff. This and other information was regularly recorded on health monitoring sheets which were also used to record the dates of any relevant events, such as holidays or hospitalisations, in the lives of participants.

\section{Results}

\section{Vignette - emergency hospitalisation}

We explored sensor firings at the time of emergency hospitalisation of one participant, Miss E. The two-week hospitalisation took place in March 2006 and was caused by a sudden rise in her blood pressure. Miss E was 84 years old and lived alone in a small flat. She had both vision and hearing impairment, epilepsy and a history of coronary heart disease. Sixteen sensors were installed in her flat (see Figure 1). In the period before her emergency hospitalisation, a slight increase in her blood pressure was recorded on her health monitoring sheets. Nevertheless, until the day of the crisis, all her blood pressure readings were within the range considered normal for her.

We retrospectively observed a number of changes in sensor firings in the period leading towards her hospitalisation compared with the period after her return from hospital. Before hospitalisation there was more bed sensor activity during the night time, early morning hours and the time of the afternoon naps (see Figures 2 and 3), more chair sensor firings during the night time and fewer during the day time, and an increase in activity of the bathroom movement sensor, fridge door contact sensor and electric usage sensor on the kettle during the night time.

All these changes seemed to correspond well with the interpretation that Miss E was feeling increasingly unwell in the period leading up to her hospitalisation and that her sleep pattern and overall wellbeing improved after she returned from hospital. Subsequently she confirmed our interpretation during the interview.

\section{Discussion}

The present study exposed a number of limitations of the lifestyle monitoring system employed in the project. First, it exposed a weakness in the information provided by the lifestyle monitoring devices. Our triangulation exercise showed that such information 
cannot stand on its own. In order to interpret the data provided by the devices, one needs rich contextual information which is normally accumulated through social interactions between care-givers and care-receivers.

Second, the retrospective analysis of sensor firings was time consuming. In addition, some form of validation of the results has to be performed by those who are being monitored. Third, in the vignette presented above we have assumed that the pattern of sensor firings after Miss E returned from hospital was more akin to a wellbeing pattern than the pattern pre-hospitalisation. Questions remain about how to decide which pattern of sensor firings represents the norm for a particular individual, and who should makes such a decision. It is important to bear in mind that any norm is likely to change over time, which may alter the criteria used for raising alerts.

Overall, the present study suggests that the availability of contextual information about the events and everyday activities of participants is important both at the stage of choosing appropriate lifestyle monitoring sensors and when attempting to provide a meaningful interpretation of their results. Obtaining such information will depend on establishing a rapport and continuous communication with participants and their carers.

\section{Acknowledgements}

The study was funded by the EPSRC EQUAL (grant no. GR/S29058/01). We thank the research participants and the housing scheme's staff.

\section{References}

1 Brownsell S., Bradley D. Assistive Technology and Telecare. Forging Solutions for Independent Living. Bristol: Policy Press, 2003 
Table 1 Sensors installed in the flats

\begin{tabular}{|l|c|c|c|c|c|c|c|}
\hline Sensors & Mrs A* & Mrs B & Miss E & Mr G & Mr H & Mrs J & Total \\
\hline Bed-occupancy & 1 & 0 & 1 & 1 & 0 & 1 \\
\hline Chair-occupancy & 2 & 0 & 1 & 0 & 0 & 4 \\
\hline Door-usage & 4 & 4 & 4 & 4 & 7 & 1 \\
\hline Electrical-usage & 3 & 4 & 3 & 4 & 4 & 4 \\
\hline Movement (PIRs) & 5 & 6 & 5 & 5 & 5 & 27 \\
\hline \multicolumn{1}{r|}{ Total } & 15 & 14 & 14 & 14 & 16 & 6 \\
\hline
\end{tabular}




\section{Figure legends}

1. Position of the sensors in Miss E's flat

2. 24-hour pattern of bed sensor firings before hospitalisation (showing total hourly firings over 37 days)

3. 24-hour pattern of bed sensor firings after hospitalisation (showing total hourly firings over 37 days)

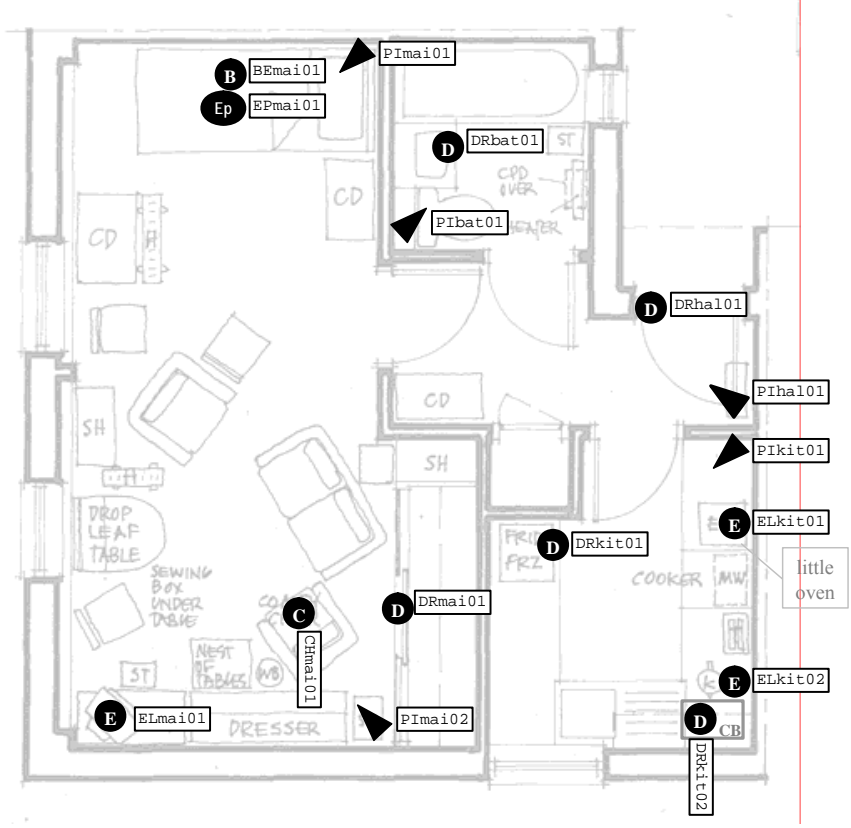



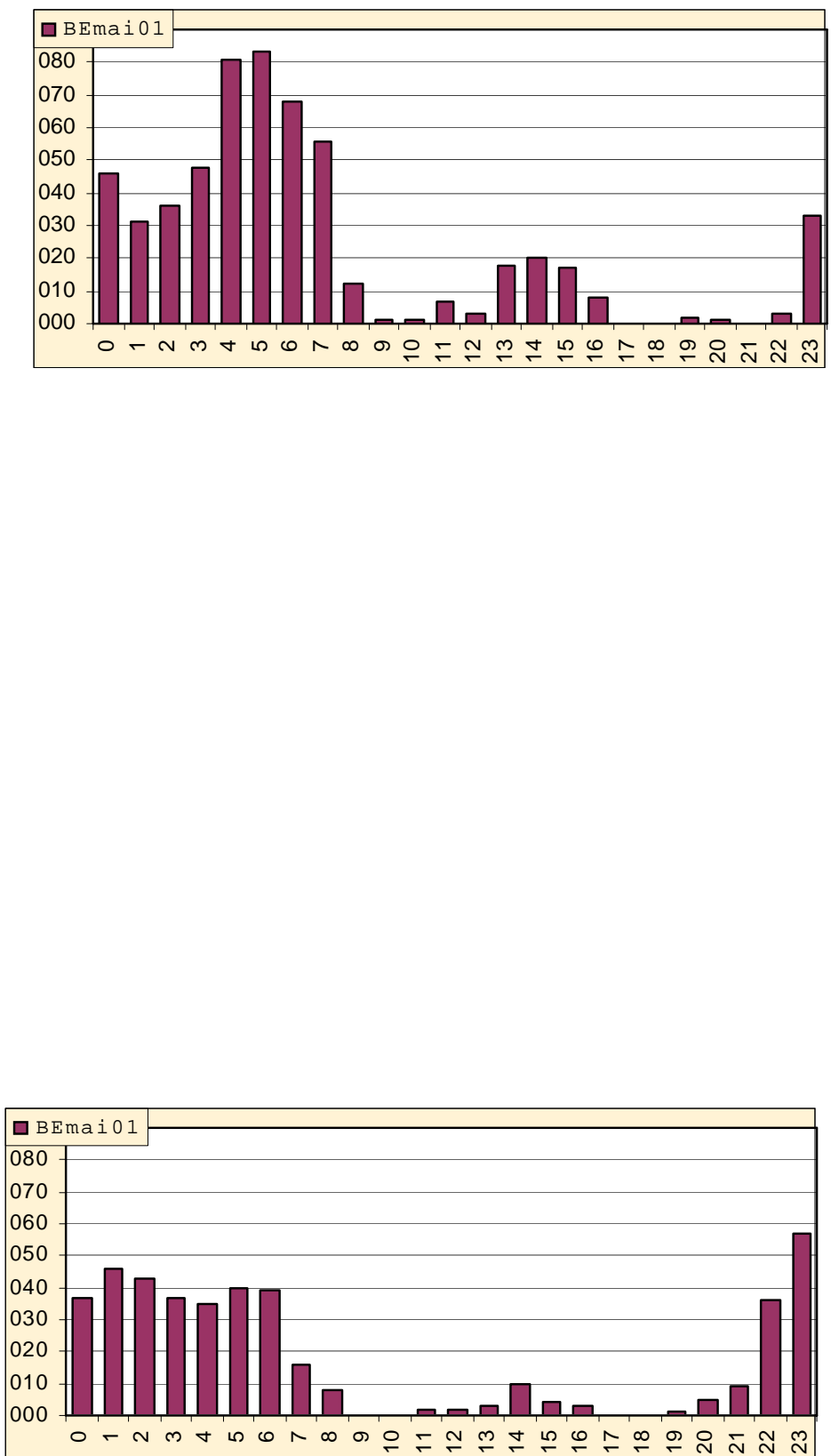